\title{
Translation of Ser ${ }^{16}$ and Thr $^{17}$ phosphorylation of phospholamban into $\mathrm{Ca}^{2+}$-pump stimulation
}

\author{
Wayne A. JACKSON and John COLYER ${ }^{\star}$ \\ Department of Biochemistry and Molecular Biology, University of Leeds, Leeds LS2 9JT, U.K.
}

Stimulation of cardiac sarcoplasmic reticulum $\mathrm{Ca}^{2+}$-pump activity is achieved by phosphorylation of the oligomeric protein phospholamban at either $\operatorname{Ser}^{16}$ or $\mathrm{Thr}^{17}$. The altered mobility of phosphorylated forms of pentameric phospholamban has been utilized to demonstrate that the mechanisms of phosphorylation of the two sites differ. Phosphorylation of $\mathrm{Ser}^{16}$ by the AMPdependent protein kinase proceeds via a random mechanism $[\mathrm{Li}$, Wang and Colyer (1990) Biochemistry 29, 4535-4540], whereas phosphorylation of $\mathrm{Thr}^{17}$ by calmodulin-dependent protein kinase is shown here to proceed via a co-operative mechanism. This co-operative reaction mechanism was unaffected by the phosphorylation status of Ser ${ }^{16}$. These two mechanisms of phosphorylation generate very different phosphoprotein profiles depending on whether the $\operatorname{Ser}^{16}$ or $\mathrm{Thr}^{17}$ residue is phosphorylated. The translation of these patterns of phosphorylation into $\mathrm{Ca}^{2+}$ pump function was reviewed using a fluorimetric $\mathrm{Ca}^{2+}$-indicator dye, fluo-3, to measure $\mathrm{Ca}^{2+}$ uptake by cardiac sarcoplasmic reticulum vesicles. The rate of $\mathrm{Ca}^{2+}$ accumulation, which parallels $\mathrm{Ca}^{2+}$-pump activity, was stimulated in proportion with the stoichiometry of phospholamban phosphorylation, irrespective of whether phosphorylation was on $\mathrm{Ser}^{16}$ or $\mathrm{Thr}^{17}$.

\section{INTRODUCTION}

The regulation of sarcoplasmic reticulum (SR) $\mathrm{Ca}^{2+}$-pump activity by phospholamban (PLB) is an important control feature of the homoeostasis of $\mathrm{Ca}^{2+}$ in cardiac muscle. $\mathrm{Ca}^{2+}$ ions represent the molecular switch for muscle contraction, and much of the $\mathrm{Ca}^{2+}$ required for this purpose is mobilized from the $\mathrm{SR}$ in response to an action potential, and returns there to facilitate relaxation. The rate at which $\mathrm{Ca}^{2+}$ is sequestered by the $\mathrm{SR}$ can be modulated by circulating catecholamines, and this modification contributes to the positive inotropic and chronotropic responses of these agents. Catecholamines modify $\mathrm{Ca}^{2+}$ handling primarily through the phosphorylation of PLB. The importance of PLB in the control of cardiac contractility was illustrated recently by the deletion of the PLB gene in transgenic mice [1]. Homozygous $\mathrm{PLB}^{-}$mice display cardiac contractile parameters equivalent to $\mathrm{PLB}^{+}$littermates exposed to the synthetic $\beta$-agonist, isoprenaline [1]. An equivalent conclusion was made from an earlier study in which monoclonal antibodies to PLB were introduced into cardiac myocytes. The $\mathrm{Ca}^{2+}$-handling properties of such cells were stimulated such that a $\beta$-agonist could offer no further alteration in the transient [2]. Thus the control of the phosphorylation status of PLB is a major contributor to the contractile status of cardiac muscle.

PLB is a small type-II membrane protein (52 amino acids [3]) associated with the SR/endoplasmic-reticulum $\mathrm{Ca}^{2+}$-ATPase (SERCA2) of cardiac muscle, smooth muscle and slow-twitch skeletal muscle [4]. PLB is normally a repressor of $\mathrm{SR} \mathrm{Ca}^{2+}$-pump function, but phosphorylation by cAMP-dependent protein kinase (PKA) (of $\mathrm{Ser}^{16}$ exclusively $[5,6]$ ) or by calmodulin (CaM)dependent protein kinase (of $\operatorname{Thr}^{17}$ exclusively $[5,6]$ ) reverses this effect. The relative importance of $\mathrm{Ser}^{16}$ and $\mathrm{Thr}^{17}$ phosphorylation in the modulation of cardiac contractility has been difficult to assess. Both sites contribute to the increased $\mathrm{Ca}^{2+}$-sequestration rate on $\beta$-agonist exposure in vivo [7], although the usage of these two sites appears to vary with species [8]. In terms of $\mathrm{Ca}^{2+}$-pump enzymology, both $\mathrm{Ser}^{16}$ phosphorylation [9] and $\mathrm{Thr}^{17}$ phosphorylation produce $\mathrm{Ca}^{2+}$-pump stimulation [10]; however, phosphorylation of both sites within a single PLB polypeptide does not result in double stimulation of $\mathrm{Ca}^{2+}$-pump function [9]. The effects may appear additive in terms of $\mathrm{Ca}^{2+}$ transport in vivo, if the population of PLB phosphorylated on Ser ${ }^{16}$ differs from that phosphorylated on $\mathrm{Thr}^{17}$, as has been suggested previously [11]. At a molecular level, the interaction of PLB and the $\mathrm{Ca}^{2+}$ pump is known to involve residues 2-18 of the cytoplasmic domain of PLB [12] and 397-402 [13] and elements within 467-762 [14] of SERCA2. This interaction is further complicated by the oligomeric arrangement of PLB (pentamer) which has been defined in SDS $[5,15,16]$, and which we have argued previously predominates in native SR membranes [17]. In native SR membranes a number of $\mathrm{Ca}^{2+}$-pump units may interact with each pentamer of PLB. In an effort to determine the number of $\mathrm{Ca}^{2+}$ pumps likely to be co-ordinated around a single pentamer of PLB, the relative concentration of the two proteins has been determined in SR membranes and in reconstitution studies. Good agreement exists between these two studies which describe a molar ratio of $2: 1 \mathrm{PLB} / \mathrm{ATPa} e$ in SR membranes [9], and 3:1 in reconstitution systems where maximal $\mathrm{Ca}^{2+}$-ATPase control had been achieved [18]. These data are consistent with the idea that two $\mathrm{Ca}^{2+}$ pumps interact with separate subunits of PLB within a PLB homopentamer as depicted in Figure 1.

The oligomeric structure of PLB does not appear to be essential for biological function [12], but two potential benefits of the tertiary structure might be (i) to facilitate a co-operative mechanism of phosphorylation and dephosphorylation of the protein, and/or (ii) to translate low levels of pentamer phosphorylation into large increases in $\mathrm{Ca}^{2+}$-pump function [9]. To investigate these possibilities, a preliminary investigation of the mechanism of $\mathrm{Ca}^{2+}$-pump stimulation by $\mathrm{Ser}^{16}$ phosphorylation has been reported [9], and the data presented were consistent

Abbreviations used: CaM, calmodulin; PKA, CAMP-dependent protein kinase; PKI, peptide inhibitor of PKA; PLB, phospholamban; SR, sarcoplasmic reticulum; SERCA2, SR/endoplasmic-reticulum Ca ${ }^{2+}$-ATPase; ATP[S], adenosine 5'-[ $\gamma$-thio]triphosphate.

* To whom correspondence should be addressed. 


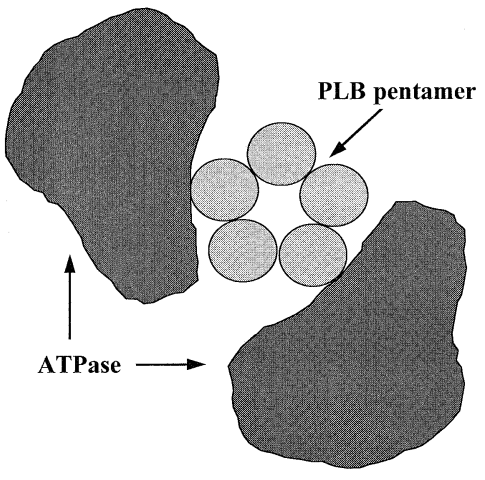

Figure 1 Schematic representation of PLB-Ca ${ }^{2+}$-ATPase complex

View perpendicular to the bilayer surface using $\mathrm{Ca}^{2+}$-ATPase and transmembrane helical dimensions from ref [19] assuming the protein ratio described in ref. [9].

with three theoretical models, in which $\mathrm{Ca}^{2+}$-pump stimulation was: (i) proportional to phosphorylation stoichiometry; (ii) a threshold event triggered by the generation of tris-phosphorylated pentamer; or (iii) stimulated in a 'delayed linear' manner, i.e. proportional to phosphorylation stoichiometry beyond a critical value (1 subunit/pentamer). The objective of this current study is to define the translation of $\operatorname{Ser}^{16}$ and $\mathrm{Thr}^{17}$ phosphorylation of PLB into $\mathrm{Ca}^{2+}$-pump function. This first required description of the mechanism of $\mathrm{Thr}^{17}$ phosphorylation as had previously been performed for $\operatorname{Ser}^{16}$ phosphorylation [16]. We demonstrate that the CaM kinase II endogenous to SR vesicles exploits the close proximity of five phosphorylation sites within a pentameric unit of PLB and exercises a co-operative reaction mechanism of phosphorylation. This results in two distinct pools of PLB: pentamers that are fully phosphorylated and those that are fully dephosphorylated; a pattern of phosphorylation species radically different from that achieved during $\mathrm{Ser}^{16}$ phosphorylation [16]. The translation of these two distinctive patterns of PLB phosphorylation into $\mathrm{Ca}^{2+}$-pump stimulation was shown to be simple, with pump activity increasing in proportion to phosphorylation stoichiometry in each case.

\section{EXPERIMENTAL}

\section{Materials}

Canine cardiac SR vesicles were isolated as described previously [16]. CaM was purified from bovine testes by the method of Gopalakrishna and Anderson [20]. The catalytic subunit of PKA was purified as described previously [21]. The monoclonal antibody raised against canine cardiac PLB, A1 [22], was purified by Protein G chromatography.

\section{Thiophosphorylation of PLB}

\section{$\mathrm{Thr}^{17}$ thiophosphorylation by CaM-dependent protein kinase}

SR vesicles were thiophosphorylated at a concentration of $1 \mathrm{mg} / \mathrm{ml}$ by incubating them at $10^{\circ} \mathrm{C}$ in $50 \mathrm{mM}$ histidine $/ \mathrm{HCl}$, $\mathrm{pH} 7$, containing $5 \mathrm{mM} \mathrm{MgSO}, 2 \mu \mathrm{M} \mathrm{CaM}, 1 \mu \mathrm{M}$ PKA inhibitor peptide (PKI, 5-22 amide [23]), $0.2 \mathrm{mM}$ adenosine $5^{\prime}$-[ $\gamma$-thio] triphosphate (ATP[S]) and $1 \mu \mathrm{M}$ Microcystin-LR. The $\mathrm{Ca}^{2+}$ required for this reaction was that which contaminated our buffer solutions $(\sim 1 \mu \mathrm{M})$. Samples were removed over a $30 \mathrm{~min}$ time course, and the reaction was terminated by snap-freezing in liquid $\mathrm{N}_{2}$. The samples were either used in fluo-3-based $\mathrm{Ca}^{2+}$ uptake assays (below) or were thawed in the presence of SDS sample buffer in order to determine the mechanism of phosphorylation by subjecting the samples to SDS/PAGE on $15 \%$ polyacrylamide gels [24] and immunoblotting on to poly(vinylidene difluoride) membrane using a semidry blotting apparatus (Pharmacia). The membranes were stained for PLB using a specific monoclonal antibody, A1, as described previously [16]. The electrophoretic separation of PLB phosphorylation species was used to determine the stoichiometry of thiophosphorylation by the method of Colyer and Wang [9].

\section{$\operatorname{Ser}^{16}$ thiophosphorylation by PKA}

SR vesicles were thiophosphorylated at a concentration of $1 \mathrm{mg} / \mathrm{ml}$ by incubating them at $30^{\circ} \mathrm{C}$ in $50 \mathrm{mM}$ histidine $/ \mathrm{HCl}$, $\mathrm{pH} 7$, containing $5 \mathrm{mM} \mathrm{MgSO}_{4}, 0.2 \mathrm{mM} \mathrm{ATP}[\gamma \mathrm{S}]$ and $1 \mu \mathrm{M}$ Microcystin-LR, and various concentrations of PKA (50$500 \mathrm{nM}$ ). Samples were removed after various incubation times in order to achieve particular thiophosphorylation stoichiometries, and the reaction was terminated after the addition of $1 \mu \mathrm{M}$ PKI by snap-freezing in liquid $\mathrm{N}_{2}$. The samples were used in fluo-3-based $\mathrm{Ca}^{2+}$-uptake assays, and an aliquot of each was used to determine the thiophosphorylation stoichiometry [9].

\section{Dual thiophosphorylation of PLB}

$\mathrm{SR}$ vesicles were thiophosphorylated at a concentration of $1 \mathrm{mg} / \mathrm{ml}$ by incubating them at $30^{\circ} \mathrm{C}$ in $50 \mathrm{mM}$ histidine $/ \mathrm{HCl}$, $\mathrm{pH} 7$, containing $5 \mathrm{mM} \mathrm{MgSO}{ }_{4}, 0.2 \mathrm{mM}$ ATP $[\gamma \mathrm{S}], 100 \mathrm{nM}$ PKA and $1 \mu \mathrm{M}$ Microcystin-LR for $10 \mathrm{~min}$. PKA activity was terminated by the addition of $1 \mu \mathrm{M}$ PKI, and the endogenous CaMdependent protein kinase activity was stimulated simultaneously by the addition of $2 \mu \mathrm{M} \mathrm{CaM}$. The $\mathrm{Ca}^{2+}$ required for this reaction was that which contaminated our buffer solutions $(\sim 1 \mu \mathrm{M})$. Samples were removed over a 30 min time course, and the reaction terminated by snap-freezing in liquid $\mathrm{N}_{2}$. The samples were thawed in the presence of SDS sample buffer [24], and the mechanism of phosphorylation was determined as described by Colyer and Wang [9].

\section{Control of the endogenous PKA-like kinase of SR}

Purified cardiac SR vesicles were incubated at a concentration of $0.5 \mathrm{mg} / \mathrm{ml}$ for $10 \mathrm{~min}$ at $30^{\circ} \mathrm{C}$ in $50 \mathrm{mM}$ histidine $/ \mathrm{HCl}, \mathrm{pH} 7$, containing $5 \mathrm{mM} \mathrm{MgSO}_{4}, 25 \mathrm{mM} \mathrm{NaF}, 1 \mathrm{mM}$ vanadate, $1 \mathrm{mM}$ EGTA, $0.2 \mathrm{mM}\left[\gamma^{\left.-{ }^{32} \mathrm{P}\right] \mathrm{ATP}}(\sim 100 \mathrm{c.p.m} . / \mathrm{pmol})\right.$ and various concentrations of PKI. Phosphorylation was terminated by the addition of SDS sample buffer [24]. The samples were boiled for $2 \mathrm{~min}$ and subjected to SDS/PAGE, using $15 \%$ polyacrylamide gels [24]. The level of phosphorylation in each sample was determined by autoradiography of the dried gels, and quantified by liquid-scintillation counting of pieces of gel which contained PLB.

\section{Control of the endogenous phosphatase of SR}

Purified cardiac SR vesicles were incubated at a concentration of $0.5 \mathrm{mg} / \mathrm{ml}$ for $30 \mathrm{~min}$ at $30{ }^{\circ} \mathrm{C}$ in $50 \mathrm{mM}$ histidine $/ \mathrm{HCl}, \mathrm{pH} 7$, containing $5 \mathrm{mM} \mathrm{MgSO}_{4}, 0.5 \mu \mathrm{M}$ PKA and $0.2 \mathrm{mM} \mathrm{ATP}[\mathrm{S}]$. The phosphorylation was terminated by the addition of $1 \mu \mathrm{M}$ PKI and the SR vesicles were incubated in the presence or absence of $3.5 \mathrm{mM}$ sodium pyrophosphate for $30 \mathrm{~min}$ at $30^{\circ} \mathrm{C}$. Samples were removed at $5 \mathrm{~min}$ intervals over this time course and the reaction was terminated by the addition of SDS sample buffer [24]. The phosphorylation status of PLB in all the samples was determined as described by Colyer and Wang [9]. 


\section{$\mathrm{Ca}^{2+}$-uptake assay}

$\mathrm{Ca}^{2+}$ uptake by purified SR vesicles was followed using a fluo-3based assay as described previously [25]. SR vesicles (100 $\mu \mathrm{g}$ of protein) were incubated at $37^{\circ} \mathrm{C}$ with $10 \mathrm{mM}$ potassium phosphate, $\mathrm{pH} 7$, containing $3.5 \mathrm{mM}$ sodium pyrophosphate, $120 \mathrm{mM} \mathrm{KCl}, 10 \mathrm{mM}$ phosphocreatine, $10 \mu \mathrm{g} / \mathrm{ml}$ creatine kinase (8 units/ml), $250 \mathrm{nM}$ fluo-3 (free acid, cell impermeant), $1 \mu \mathrm{M}$ PKI and, in some experiments, $200 \mathrm{nM}$ staurosporin. $\mathrm{Ca}^{2+}$ uptake was initiated after $5 \mathrm{~min}$ by the addition of $1.5 \mathrm{mM} \mathrm{MgATP}$, and monitored fluorimetrically (excitation $506 \mathrm{~nm}$, emission $526 \mathrm{~nm}$, slits $5 \mathrm{~nm}$ ). Minimum and maximum fluorescence values were obtained for each assay by the sequential addition of $1 \mathrm{mM}$ EGTA and $3.5 \mathrm{mM} \mathrm{CaCl} \mathrm{Cl}_{2}$ respectively. Fluorescence data were converted into extravesicular $\mathrm{Ca}^{2+}$ concentration using the equation:

$\left[\mathrm{Ca}^{2+}\right]=K_{\mathrm{d}}\left(F-F_{\min }\right) /\left(F_{\max }-F\right)$

where $F_{\min }$ and $F_{\max }$ are the minimum and maximum fluorescence values respectively for each assay, and the $K_{\mathrm{d}}$ is the $\mathrm{Ca}^{2+}$ dissociation constant for fluo-3 (determined to be $320 \mathrm{nM}$ in our hands, using binding constants described in [26]). The rate of $\mathrm{Ca}^{2+}$ uptake was then determined by taking the tangent to the uptake data at the extravesicular $\left[\mathrm{Ca}^{2+}\right]$ of interest. Maximum stimulation of $\mathrm{Ca}^{2+}$-uptake activity generated by inclusion of monoclonal antibody (A1 [27]) was established by incubation of the SR sample $(100 \mu \mathrm{g})$ with A1 $(150 \mu \mathrm{g})$ for $5 \mathrm{~min}$ on ice before transfer into the assay solution.

\section{RESULTS AND DISCUSSION}

\section{Thr ${ }^{17}$ phosphorylation of PLB}

The oligomeric nature of PLB presents an opportunity for cooperation in the mechanism of phosphorylation and dephos- phorylation. The phosphorylation of one subunit within a pentamer may influence the probability of phosphorylation of the others within that structure. This can be studied as a consequence of the electrophoretic separation of pentameric forms of PLB as a function of the number of subunits phosphorylated [17], which has been used previously to demonstrate that $\operatorname{Ser}^{16}$ phosphorylation by PKA is a random event whereas dephosphorylation of that phosphoprotein is strictly co-operative [16]. To investigate the mechanism of control of $\mathrm{Ca}^{2+}$-pump function reversed by PKA-dependent $\left(\mathrm{Ser}^{16}\right)$ or $\mathrm{CaM}$-dependent phosphorylation $\left(\mathrm{Thr}^{17}\right.$ ) of PLB, we sought to establish first the mechanism of phosphorylation of $\mathrm{Thr}^{17}$, and then to establish the translation of both of these events into $\mathrm{Ca}^{2+}$-pump stimulation.

Figure 2 depicts the phosphorylation of PLB (pentamer displayed) by a CaM-dependent protein kinase endogenous to the SR. Phosphorylation was completely dependent on $\mathrm{Ca}^{2+} / \mathrm{CaM}$ and resulted in the exclusive phosphorylation of $\mathrm{Thr}^{17}$ (confirmed by using phosphoamino acid analysis [28] and phosphorylation-state-specific antibodies to PLB [29], data not shown). A change in the mobility of PLB accompanied phosphorylation, as has been demonstrated previously [16], which facilitated description of the thiophosphorylation of PLB to a stoichiometry of $\sim 2.5 \mathrm{~mol} / \mathrm{mol}$ of pentamer, accompanied by a distinctive pattern of phosphorylation products in which $\mathrm{P}_{5}$ pentamers (all five subunits phosphorylated) accumulated without a preceding accumulation of pentamers phosphorylated on fewer subunits $\left(\mathrm{P}_{1}-\mathrm{P}_{4}\right)$. The concentration of pentamers phosphorylated on an intermediate number of subunits $\left(\mathrm{P}_{1}-\mathrm{P}_{4}\right)$ remained low and constant throughout the reaction (Figure 2B). This is indicative of a co-operative reaction mechanism in which phosphorylation of the first subunit within a pentamer promotes the phosphorylation of all others within that unit. Comparison of the experimental distribution of phosphorylated species of
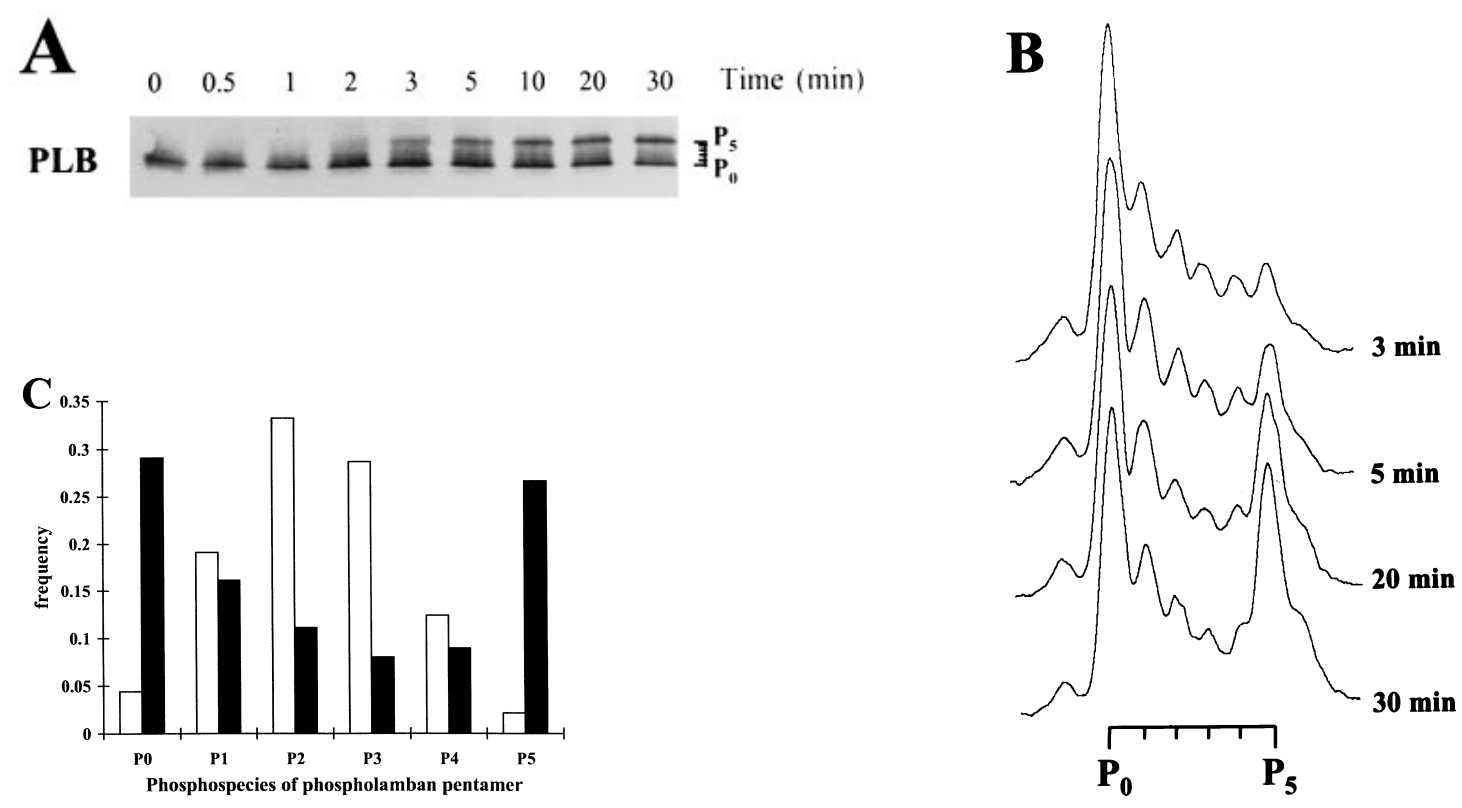

Figure 2 Time course of thiophosphorylation of PLB by CaM-dependent protein kinase endogenous to the SR

SR vesicles were thiophosphorylated by the endogenous CaM-dependent protein kinase activity as described in the Experimental section. (A) Immunoblot showing the oligomeric form of PLB with the phosphorylation species identified $\left(\mathrm{P}_{0}-\mathrm{P}_{5}\right.$ respectively). (B) Denisitometric analysis of the oligomeric species of PLB phosphorylated for various times. (C) Comparison of the relative concentration of individual phosphorylation species of PLB after 20 min thiophosphorylation ( $\mathbf{a}$ ) with theoretical prediction by the binomial equation at this point in stoichiometry (2.32 mol/mol of pentamer), (口). 


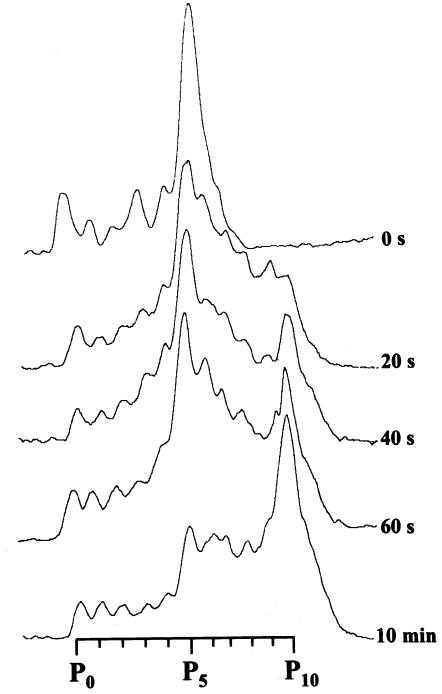

Figure 3 Phosphorylation of $\mathrm{Thr}^{17}$ after prior phosphorylation of $\mathrm{Ser}^{16}$

Sequential phosphorylation of PLB by PKA and CaM-dependent protein kinase was performed as described in the Experimental section. The densitometric analysis of the oligomeric species of PLB resolved by SDS/PAGE and immunoblotting is presented.

PLB $\left(\mathrm{P}_{0}-\mathrm{P}_{5}\right)$ with mathematical predictions based on a random mechanism [16], (Figure 2C) for the $20 \mathrm{~min}$ time point clearly demonstrates that $\mathrm{Thr}^{17}$ phosphorylation is not a random event, but proceeds by a co-operative mechanism. The co-operative nature of this reaction is significant for two reasons: first it provides evidence that the oligomeric form of PLB is present in the isolated SR membrane (for CaM kinase II to phosphorylate); and secondly it generates a pattern of phosphorylation species distinct from those generated by Ser $^{16}$ phosphorylation [16]. An understanding of the translation of these divergent phosphorylation reactions into $\mathrm{Ca}^{2+}$-pump function is clearly important.

Before considering the translation of this distinctive pattern of PLB phosphorylation into $\mathrm{Ca}^{2+}$-pump stimulation, we performed a further study to determine whether the co-operative phosphorylation reaction was likely to occur in vivo. Exposure of cardiac muscle to a $\beta$-agonist results in the phosphorylation of both $\mathrm{Ser}^{16}$ and $\mathrm{Thr}^{17}$, with the phosphate incorporation into Ser ${ }^{16}$ preceding that of $\mathrm{Thr}^{17}$ [30]. As such the effects of prior $\operatorname{Ser}^{16}$ phosphorylation on the mechanism of $\mathrm{Thr}^{17}$ phosphorylation was investigated. Figure 3 shows the densitometric analysis of an experiment in which PLB was thiophosphorylated on $\mathrm{Ser}^{16}$ to a point close to full stoichiometry (zero time point of $\mathrm{CaM}$ kinase phosphorylation). At this point further Ser ${ }^{16}$ phosphorylation was inhibited by the addition of $\mathrm{PKI}$, and $\mathrm{Thr}^{17}$ thiophosphorylation initiated by the activation of the SR CaM kinase II. As was evident in the previous experiments, phosphorylation of $\mathrm{Thr}^{17}$ was a co-operative reaction with the accumulation of pentamers in which all five subunits were phosphorylated (on both $\operatorname{Ser}^{16}$ and $\mathrm{Thr}^{17}, \mathrm{P}_{10}$ ) without the accumulation of forms with a lower phosphorylation status. The co-operative phosphorylation of PLB pentamers is a robust feature of $\mathrm{Thr}^{17}$ phosphorylation, unaffected by the phosphorylation status of the neighbouring $\operatorname{Ser}^{16}$ residue.

The divergent phosphoprotein profile obtained with $\operatorname{Ser}^{16}$ and $\mathrm{Thr}^{17}$ phosphorylation of PLB may have distinct properties when translated into $\mathrm{Ca}^{2+}$-pump function. During $\mathrm{Ser}^{16}$ phosphoryl- ation, the entire PLB pool becomes phosphorylated progressively as the stoichiometry rises. With $\mathrm{Thr}^{17}$ phosphorylation, however, two pools of PLB pentamer are generated, one that is completely dephosphorylated and one that is completely phosphorylated. At low stoichiometry the former pool is of greater size than the latter, with the ratio shifting as the phosphorylation stoichiometry rises. The interpretation of each of these two patterns of PLB phosphorylation by the $\mathrm{Ca}^{2+}$ pump is of interest since it will allow extrapolation from phosphorylation data in vivo to SR functional parameters. A previous attempt to delineate this issue for Ser $^{16}$ phosphorylation was successful in part [9] and identified three theoretical solutions for the experimental observations. These were consistent with $\mathrm{Ca}^{2+}$-pump stimulation (i) proportional to phosphorylation stoichiometry, (ii) being a threshold event triggered by the generation of a tris-phosphorylated pentamer, or (iii) stimulated in a delayed-linear manner, i.e. proportional to phosphorylation stoichiometry beyond a critical value (1 subunit/pentamer). Resolution of which of these three alternatives is correct will require the collection of $\mathrm{Ca}^{2+}$-transport data of greater accuracy and reproducibility than was achieved previously [9]. To this end, we have employed a fluorimetric $\mathrm{Ca}^{2+}$-uptake assay, using fluo-3 to monitor $\mathrm{Ca}^{2+}$-pump activity. In this assay, extravesicular $\mathrm{Ca}^{2+}$ is monitored continuously by the dye, and the rate of $\mathrm{Ca}^{2+}$ uptake by the SR vesicles can be determined at any extravesicular $\mathrm{Ca}^{2+}$ concentration from the tangent to the uptake curve at that point. This assay therefore eliminates one source of error present in alternative $\mathrm{Ca}^{2+}$-transport assays, namely those associated with $\mathrm{Ca}^{2+} /$ EGTA additions, employed to achieve calculated pCa.

Figure 4(A) shows a composite graph which characterizes the assay. On addition of MgATP, uptake of $\mathrm{Ca}^{2+}$ by the SR vesicles is observed, a process that can be halted by inhibition of pump function (thapsigargin [31], which is followed by the leakage of $\mathrm{Ca}^{2+}$ from the vesicles) or by addition of a $\mathrm{Ca}^{2+}$ ionophore (A23187). The net uptake of $\mathrm{Ca}^{2+}$ measured in this assay clearly represents a balance between $\mathrm{Ca}^{2+}$ uptake and release. If the assay is to be used to determine $\mathrm{Ca}^{2+}$-pump parameters, then the component of $\mathrm{Ca}^{2+}$ release needs to be unaffected by the phosphorylation status of PLB. Figure 4(B) shows the $\mathrm{Ca}^{2+}$ release properties of SR vesicles in which PLB phosphorylated on $\mathrm{Ser}^{16}$ to four different stoichiometries. In these experiments $\mathrm{Ca}^{2+}$ uptake into the SR was followed by $\mathrm{Ca}^{2+}$ release once ATPase activity was inhibited (250 nM thapsigargin). The rate of $\mathrm{Ca}^{2+}$ release was unaffected by phosphorylation of PLB. We are thus convinced that net $\mathrm{Ca}^{2+}$ uptake in this assay provides a sensitive and reliable index of $\mathrm{Ca}^{2+}$-ATPase function. Similar arguments are required to validate the use of ${ }^{45} \mathrm{Ca}^{2+}$-uptake parameters as a measure of $\mathrm{Ca}^{2+}$-pump function, which has gained wide support $[27,32,33]$.

\section{Control of SR kinase and phosphatase activities}

In order to investigate the relationship between the phosphorylation status of PLB (at each of the two sites) and $\mathrm{Ca}^{2+}$-pump activity, the latter needs to be measured at a range of PLB thiophosphorylation stoichiometries. The stoichiometry of phosphorylation must remain constant during the time course of the assay for the measurement to be useful, and thus any endogenous kinase or phosphatase activity must be removed. The SR has been shown to contain an endogenous type-1 phosphatase [34] along with both PKA-like [16] and CaMdependent kinase [35] activities.

For SR vesicle samples phosphorylated by PKA $\left(\operatorname{Ser}^{16}\right)$, only the phosphatase and PKA-like activities are active. In the absence of CaM (as is the case here) the endogenous CaM kinase activity 

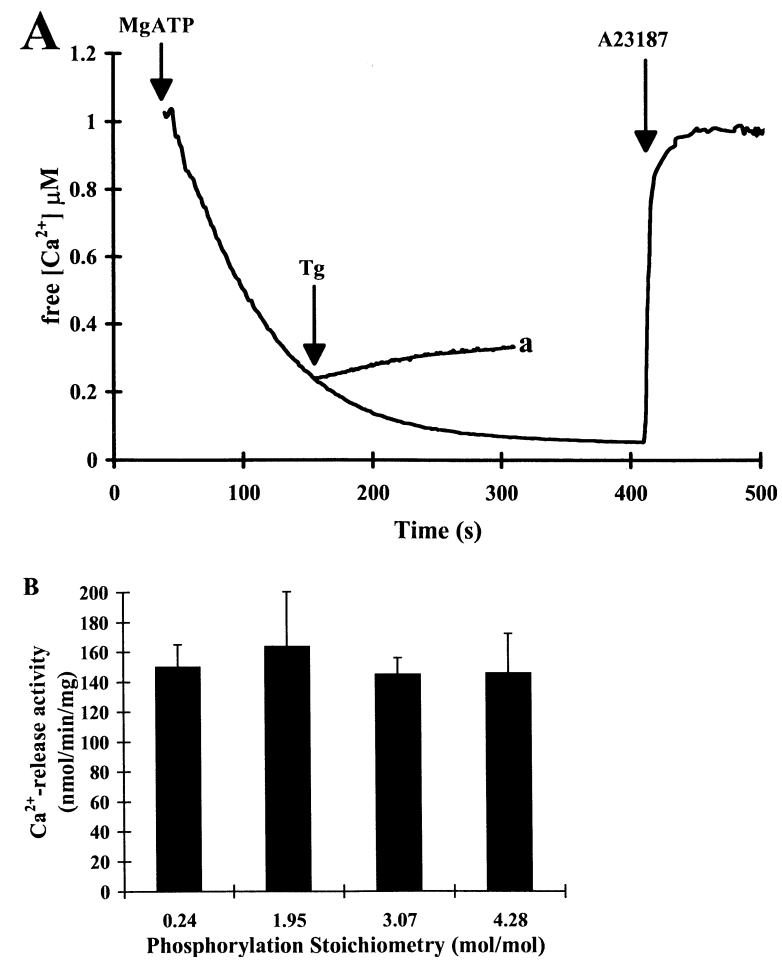

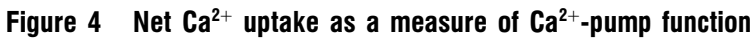

$\mathrm{Ca}^{2+}$ sequestration by SR vesicles was measured as described in the Experimental section. (A) Uptake was initiated by the addition of $1.5 \mathrm{mM} \mathrm{MgATP}$, and accumulated $\mathrm{Ca}^{2+}$ released by the addition of $\mathrm{A} 23187(12 \mu \mathrm{M})$. $\mathrm{Ca}^{2+}$ uptake was also inhibited by the addition of $250 \mathrm{nM}$ thapsigargin (Tg, trace a). (B) $\mathrm{Ca}^{2+}$-release rates were compared for SR vesicles phosphorylated by PKA to various stoichiometries (expressed relative to PLB pentamer). The data represent means \pm S.D. $(n=4)$. Release was promoted by the addition of $250 \mathrm{nM}$ thapsigargin at $80 \mathrm{nM} \mathrm{Ca}^{2+}$.

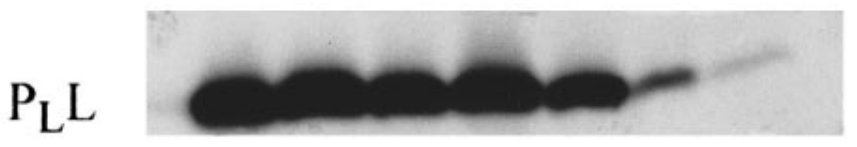

\section{PKI (nM) $\quad 0 \quad 20 \quad 50 \quad 1002005001000$}

\section{Figure 5 Control of endogenous PKA-like kinase}

PLB was phosphorylated by kinase activities endogenous to the SR in the presence and absence of peptide inhibitor PKI as described in the Experimental section. Samples were resolved by SDS/PAGE, and the autoradiograph of PLB monomer $\left(P_{L} L\right)$ is presented.

is latent. Figure 5 defines the conditions used to control the PKA-like kinase. Phosphorylation of PLB by this kinase was inhibited by the inclusion of increasing concentrations of the peptide inhibitor PKI, which is based on the PKA-specific heatstable inhibitor protein [23]. Control of the kinase was ensured at $1 \mu \mathrm{M}$ PKI. Endogenous phosphatase also required inhibition. Thiophosphorylation of PLB was performed to increase the stability of the phosphoprotein; however, this protection was only partial (Figure 6). The addition of pyrophosphate $(3.5 \mathrm{mM})$, which served as a precipitating anion in the $\mathrm{Ca}^{2+}$-uptake assay, provided effective phosphatase control (Figure 6). Thus $\mathrm{Ca}^{2+}$ uptake assays for $\mathrm{Ser}^{16}$-phosphorylated samples were performed

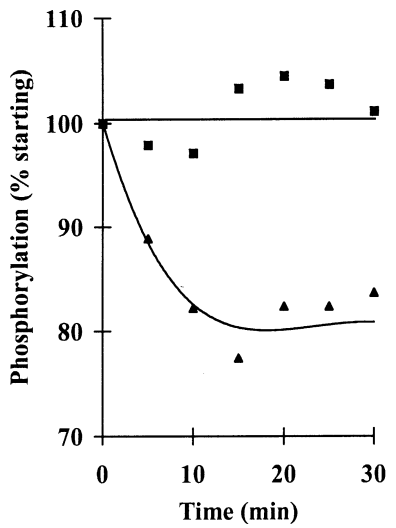

Figure 6 Control of endogenous phosphatase

SR vesicles were thiophosphorylated to $4 \mathrm{~mol} / \mathrm{mol}$. Dephosphorylation was initiated by the addition of PKI $(1 \mu \mathrm{M})$, and the time course followed in the absence $(\mathbf{A})$ or presence of $3.5 \mathrm{mM}$ pyrophosphate. Data points represent single observations, with the data set being representative of two experiments.

in the presence of $1 \mu \mathrm{M}$ PKI and $3.5 \mathrm{mM}$ pyrophosphate using thiophosphorylated SR samples to prevent alteration of phosphorylation stoichiometry during the experiment.

With $\operatorname{Thr}^{17}$ (CaM kinase)-phosphorylated SR vesicles, all three endogenous activities are potentially active because CaM is added in order for CaM kinase to be active. Control of this enzyme activity was effectively achieved by the addition of $200 \mathrm{nM}$ staurosporin (non-selective kinase inhibitor; results not shown) to the assay media. Thus $\mathrm{Ca}^{2+}$-uptake assays for $\mathrm{Thr}^{17}$ phosphorylated samples were performed in the presence of $200 \mathrm{nM}$ staurosprin, $1 \mu \mathrm{M}$ PKI and $3.5 \mathrm{mM}$ pyrophosphate using thiophosphorylated SR samples.

\section{$\mathrm{Ca}^{2+}$-pump stimulation by PLB phosphorylation}

Having established the $\mathrm{Ca}^{2+}$-uptake assay and the conditions required to maintain the phosphorylation status of PLB, SR samples were thiophosphorylated to a range of stoichiometries and the activity of the $\mathrm{Ca}^{2+}$-pump was determined. In previous work the translation of $\mathrm{Ser}^{16}$ phosphorylation into $\mathrm{Ca}^{2+}$-pump stimulation was compatible with three models of coupling [9]. These three models were most dissimilar at low phosphorylation stoichiometries, where models (ii) and (iii) (see the Introduction) both predicted little functional change in $\mathrm{Ca}^{2+}$ transport at a phosphorylation of $1 \mathrm{~mol} / \mathrm{mol}$ of pentamer whereas model (i) predicted $20 \%$ of maximal stimulation. Thus by focusing attention on this part of the relationship in particular, the mechanism of translation of PLB phosphorylation into $\mathrm{Ca}^{2}$-pump stimulation can be solved.

Multiple SR samples $(n=5)$ were thiophosphorylated by PKA ( Ser $^{16}$ site) to stoichiometries of approx. 1, 2.5 and $4 \mathrm{~mol} / \mathrm{mol}$, and the precise stoichiometry of each was determined. $\mathrm{A} \mathrm{Ca}^{2+}$-uptake assay was performed on each sample, and the data are presented as means \pm S.D. for each parameter (Figure 7A). These data demonstrate that phosphorylation of PLB is accompanied by a change in $\mathrm{Ca}^{2+}$-pump activity in direct proportion to the stoichiometry achieved. This relationship is true for all stoichiometries of Ser $^{16}$ phosphorylation, including $1 \mathrm{~mol} / \mathrm{mol}$ pentamer, and this relationship remained in effect for all measured free $\left[\mathrm{Ca}^{2+}\right]\left(63-800 \mathrm{nM} \mathrm{Ca}^{2+}\right)$. More complicated models of coupling phosphorylation to pump activity (threshold; delayed-linear mechanisms) fail to predict this event, and are 


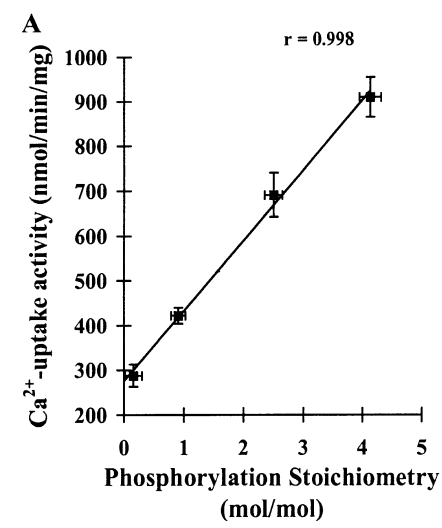

B
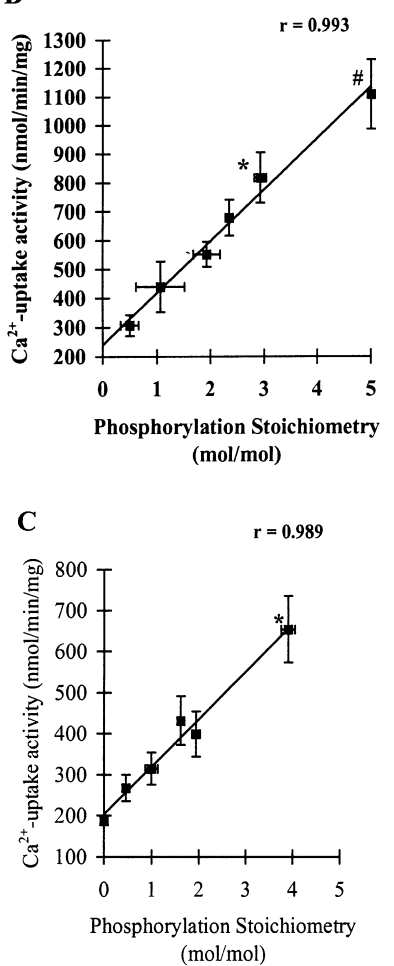

Figure 7 Dependence of $\mathrm{Ca}^{2+}$-uptake rate on $\mathrm{Ser}^{16}$ and $\mathrm{Thr}^{17}$ phosphorylation of PLB

$\mathrm{Ca}^{2+}$ uptake by SR vesicles thiophosphorylated to a range of PLB phosphorylation stoichiometries was measured using fluo-3 as described in the Experimental section. Data at $100 \mathrm{nM}$ free $\mathrm{Ca}^{2+}$ are shown for Ser ${ }^{16}$-phosphorylated SR vesicles (A) and Thr ${ }^{17}$-phosphorylated $S R$ vesicles (two separate SR preparations; B. C) (means \pm S.D. : $n=4$ ). Reference samples of monoclonal antibody A1-stimulated (\#) SR vesicles (B), and SR vesicles phosphorylated at the $\mathrm{Ser}^{16}$ residue $\left(^{\star}\right)$ of PLB $(\mathbf{B}, \mathbf{C})$ were included for the assays of $\mathrm{Th}^{17}$-phosphorylated SR vesicles. Phosphorylation stoichiometry is expressed relative to PLB pentamer. $r=$ correlation coefficient for linear regression.

thus without foundation. From these data we can conclude that, in canine cardiac SR, each phosphorylation of PLB on $\mathrm{Ser}^{16}$ contributes equally to pump stimulation.

The effect of thiophosphorylation by CaM kinase II (Thr ${ }^{17}$ site) on pump activity was then investigated. Thiophosphorylation at this site was limited to stoichiometries in the range $0-2.5 \mathrm{~mol} / \mathrm{mol}$ of pentamer, which represents the limit of exclusive $\mathrm{Thr}^{17}$ phosphorylation. Beyond this point, Ser ${ }^{16}$ phosphorylation begins to occur catalysed by an independent SR kinase. Thus to keep the system simple and review the coupling of $\mathrm{Thr}^{17}$ phosphorylation to pump activity the range of stoichiometries considered was limited to $0-2.5 \mathrm{~mol} / \mathrm{mol}$. Figures 7(B) and 7(C) display the relationship between $\mathrm{Thr}^{17}$ phosphorylation and $\mathrm{Ca}^{2+}$-pump stimulation measured by this assay using two different SR preparations. In Figure 7(B) two reference samples, PKA-phosphorylated SR $\left(\mathrm{Ser}^{16} ; 4 \mathrm{~mol} / \mathrm{mol}\right.$ pentamer; marked by asterisk) and monoclonal antibody A1 (to represent full stimulation as used previously $[9,27,32]$ ) have been included for comparison, whereas Figure $7(\mathrm{C})$ includes the former marker alone. The results show that $\mathrm{Ca}^{2+}$-pump activity was proportional to the stoichiometry of $\mathrm{Thr}^{17}$ phosphorylation of PLB. No deviation from this relationship is observed at any phosphorylation stoichiometry or at any free $\left[\mathrm{Ca}^{2+}\right](63-$ $800 \mathrm{nM} \mathrm{Ca}^{2+}$; results not shown). The slope of this relationship was indistinguishable from that linking $\operatorname{Ser}^{16}$ phosphorylation to pump activity (Figure 7A), as witnessed by comparison with the marker point, PKA-phosphorylated PLB. Thus a simple mechanism of coupling of both $\operatorname{Ser}^{16}$ and $\mathrm{Thr}^{17}$ phosphorylation of PLB to $\mathrm{Ca}^{2+}$-pump function exists, despite the considerable difference in the pattern of phosphoproteins generated in each case. The translation of $\mathrm{Thr}^{17}$ phosphorylation into $\mathrm{Ca}^{2+}$-pump function might be expected to be complicated by the phosphorylation of the $\mathrm{Ca}^{2+}$-pump per se [36]. In our experience, this phosphorylation would proceed to a very low stoichiometry $(\sim 0.005 \mathrm{~mol} / \mathrm{mol})$ under the assay conditions employed (thiophosphorylation, $\mathrm{pH} 7.0,10^{\circ} \mathrm{C}$ ) and would therefore contribute little to modulation of $\mathrm{Ca}^{2+}$ transport in these experiments.

Two comments can be made from these data. First, the simple mechanism of converting phosphorylation of PLB into $\mathrm{Ca}^{2+}$ pump activation (for both $\mathrm{Thr}^{17}$ and $\mathrm{Ser}^{16}$ [5]) justifies extrapolation from phosphorylation stoichiometry to $\mathrm{Ca}^{2+}$-pump parameters in vivo. This extrapolation carries a single caveat as a consequence of the observation that $\mathrm{Ca}^{2+}$-pump stimulation in response to phosphorylation of one site is not increased further on phosphorylation of the other residue within the same PLB polypeptide [9]. The incidence of dual phosphorylation introduces an element of redundancy into the relationship between phosphorylation and $\mathrm{Ca}^{2+}$ transport, such that the direct extrapolation from phosphorylation to activity need not be accurate in all instances. The electrophoretic mobility change associated with two-site phosphorylation (Figure $2[9,15,30]$ ) can be used to calculate the extent of dual phosphorylation and thereby correct for the redundancy in this relationship in vivo.

The second comment concerns the physical change induced by phosphorylation of PLB which results in $\mathrm{Ca}^{2+}$-pump stimulation. In many studies this is viewed as a physical dissociation of the two proteins $[37,38]$; however, the current data can be interpreted either in these terms or by an alternative model which assumes interaction of the pump with both phosphorylated and nonphosphorylated PLB; but inhibition of pump function only occurs when complexed with the latter form of PLB. In either case, if we view the pump-PLB interaction as a simple receptorligand interaction, then a linear response to changes in ligand concentration (which equates to dephosphorylated PLB concentration) would only be expected at low levels of receptor occupancy by the ligand. This suggests that at the $\mathrm{Ca}^{2+}$ concentrations considered in this study $(63-800 \mathrm{nM})$, which are of physiological significance, the pump is not saturated with PLB. As a consequence of this, pump activity is only partially inhibited and can respond to phosphorylation of PLB by an increase in $\mathrm{Ca}^{2+}$-transport rate in proportion to the stoichiometry of phosphorylation achieved.

We thank the BHF for financial support 


\section{REFERENCES}

1 Luo, W., Grupp, I. L., Harrer, J., Ponniah, S., Grupp, G., Duffy, J. J., Doetschman, T. and Kranias, E. G. (1994) Circ. Res. 75, 401-409

2 Sham, J. S. K., Jones, L. R. and Morad, M. (1991) Am. J. Physiol. 261, $\mathrm{H} 1344-\mathrm{H} 1349$

3 Fujii, J., Ueno, A., Kitano, K., Tanaka, S., Kadoma, M. and Tada, M. (1987) J. Clin. Invest. 79, 301-304

4 Fujii, J., Zarain-Herzberg, A., Willard, H. F., Tada, M. and MacLennan, D. H. (1991) J. Biol. Chem. 266, 11669-11695

5 Simmermann, H. K. B., Collins, J. H., Theibert, J. L., Wegener, A. D. and Jones, L. R. (1986) J. Biol. Chem. 261, 13333-13341

6 Tada, M. and Kadoma, M. (1989) Bioessays 10, 157-163

7 Lindemann, J. P. and Watanabe, A. M. (1985) J. Biol. Chem. 260, 4516-4525

8 Bennett, K. L., Orchard, C. H., Drago, G. A. and Colyer, J. (1995) J. Physiol. (London) 483, 20P-21P

9 Colyer, J. and Wang, J. H. (1991) J. Biol. Chem. 266, 17486-17493

10 LePeuch, C. J., Haiech, J. and Demaille, J. G. (1979) Biochemistry 18, 5150-5157

11 Gasser, J., Paganetti, P., Carafoli, E. and Chiesi, M. (1988). Eur. J. Biochem. 176, 535-541

12 Toyofuko, T., Kurzydlowski, K., Tada, M. and MacLennan, D. H. (1994) J. Biol. Chem. 269, 22929-22932

13 Toyofuko, T., Kurzydlowski, K., Tada, M. and MacLennan, D. H. (1994) J. Biol. Chem. 269, 3088-3094

14 Toyofuko, T., Kurzydlowski, K., Tada, M. and MacLennan, D. H. (1993) J. Biol. Chem. 268, 2809-2815

15 Fujii, J., Kadoma, M., Tada, M., Toda, H. and Sakiyama, F. (1986). Biochem. Biophys. Res. Commun. 138, 1044-1050

16 Li, C., Wang, J. H. and Colyer, J. (1990) Biochemistry 29, 4535-4540

17 Colyer, J. (1993) Cardiovasc. Res. 27, 1766-1771
18 Reddy, L. G., Jones, L. R., Cala, S. E., O'Brian, J. J., Tatulian, S. A. and Stokes, D. L. (1995) J. Biol. Chem. 270, 9390-9397

19 Stokes, D. L. and Green, N. M. (1990) J. Mol. Biol. 213, 529-538

20 Gopalakrishna, R. and Anderson, W. B. (1982) Biochem. Biophys. Res. Commun. 104, 830-836

21 Peters, K. A., Demaille, J. G. and Fischer, E. H. (1977) Biochemistry 16, 5691-5697

22 Suzuki, T. and Wang, J. H. (1986) J. Biol. Chem. 261, 7018-7023

23 Cheng, H.-C., Kemp, B. E., Pearson, R. B., Smith, A. J., Misconi, L., Van Patten, S. M. and Walsh, D. A. (1986) J. Biol. Chem. 261, 989-992

24 Laemmli, U. K. (1970) Nature (London) 227, 680-685

25 Michelangeli, F. (1991) J. Fluorescence 1, 203-206

26 Gould, G. W., East, J. M., Froud, R. J., McWhirter, J. M., Stefanova, H. I. and Lee, A. G. (1986). Biochem. J. 237, 217-227

27 Morris, G. L., Cheng, H-C., Colyer, J. and Wang, J. H. (1991). J. Biol. Chem. 266, 11270-11275

28 Duclos, B., Marcandier, S. and Cozzone, A. J. (1991) Methods Enzymol. 201, 10-21

29 Drago, G. A. and Colyer, J. (1994) J. Biol. Chem. 269, 25071-25077

30 Wegener, A. D., Simmerman, H. K. B., Lindemann, J. P. and Jones, L. R. (1989) J. Biol. Chem. 264, 11468-11474

31 Lytton, J., Westlin, M. and Hanley, M. R. (1991) J. Biol. Chem. 266, 17067-17071

32 Kimura, Y., Inui, M., Kadoma, M., Kijima, Y., Sasaki, T. and Tada, M. (1991) J. Mol. Cell. Cardiol. 23, 1223-1230

33 Xu, Z.-C. and Kirchberger, M. A. (1989) J. Biol. Chem. 264, 16644-16651

34 Steenaart, N. A. E., Ganim, J. R., Di Salvo, J. and Kranias, E. G. (1992) Arch. Biochem. Biophys. 293, 17-24

35 Kovacs, R. J., Nelson, M. T., Simmerman, H. K. B. and Jones, L. R. (1988) J. Biol. Chem. 263, 18364-18368

36 Hawkins, C., Xu, A and Narayanan, N. (1994) J. Biol. Chem. 269, 31198-31206

37 Voss, J., Jones, L. R. and Thomas, D. D. (1994) Biophys. J. 67, 190-196

38 James, P., Inui, M., Tada, M., Chiesi, M. and Carafoli, E. (1989) Nature (London) 342, 90-92

Received 8 September 1995/4 January 1996; accepted 22 January 1996 